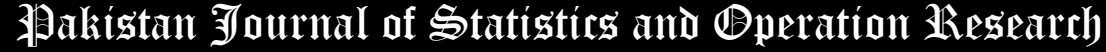

\section{New Control Charts for a Multivariate Gamma Distribution}

* Corresponding Author

\author{
Sh. Enami ${ }^{1}$, H. Torabi ${ }^{2 *}$, S.T.A. Niaki ${ }^{2}$
}

1. Department of Statistics, Yazd University, Yazd, Iran, enami1387@yahoo.com

2. Department of Statistics, Yazd University, Yazd, Iran, hamzeh.totabi@gmail.com

3. Department of Industrial Engineering, Sharif University of Technology, Tehran, Iran, niaki@ sharif.edu

\begin{abstract}
In this study, we introduce a multivariate gamma distribution, then, by defining a new statistic, three control charts called the MG charts, are proposed for this distribution. The first control chart is based on the exact distribution of this statistic, the second control chart is based on the Satterthwaite approximation, and the last is based on the normal approximation. The efficiency of the proposed control charts is evaluated by the average run length (ARL) criterion. The results show that whenever the magnitude of the parameter shifts $c<1$, the control chart based on the exact distribution has smaller ARL1s, while for $\mathrm{c}>1$, the control chart based on Satterthwaite approximation show smaller values of ARL1s. Besides, the results show that for large changes (c>2.5), all proposed charts almost have equal ARL1s; i.e. they have the same performance.
\end{abstract}

Key Words: Multivariate gamma distribution; Chi-square distribution; Average run length; Satterthwaite approximation; Normal approximation.

\section{Introduction}

Control charts play an important role in statistical quality control to monitoring and improving a production process. Sometimes researchers are interested in the investigation of more than one quality characteristic simultaneously during the production. In this case, the process is called a multivariate process.

There are many works performed in the literature in the field of control charts for multivariate attribute processes. For more discussion, one may refer to; Aslam, et.al. (2017), Ali Raza and Aslam (2018), Cozzucoli and Marozzi (2018), Chiu and Kuo (2007). Similarly, many researchers worked on various control charts in which the correlated quality characteristics are of variable type and follow some continuous probability distributions. To the best of the authors' knowledge, there is no work on the use of the multivariate gamma distribution. Nonetheless, the univariate gamma distribution has been widely used in a variety of fields; Aksoy (2000), Agarwal and Kalla (1996), Mori, et.al. (2015), Tan, et.al. (2017), to design some control charts, see for example; Aslam, et.al. (2016), Derya and Canan (2012), Khan, et.al. (2017), Zhang, et.al. (2007).

In this paper, a structure of multivariate gamma (MG) distribution will be defined. Then, by definition of a new statistic and calculating its exact distribution, a control chart for an important quality parameter of this distribution will be proposed in Section 2. In Section 3, the efficiency of this control chart will be investigated. In Section 4, the following two approximations are used to obtain the control limits:

Case 1: Satterthwaite approximation (Casella and Berger (2002));

Case 2: The Wilson-Hilferty (WH) approximation (Wilson and Hilferty (1931)).

The efficiency of the above charts in terms of the average run length (ARL) is evaluated in Section 5. ARL is the expected number of samples (subgroups) taken before there is a signal (Montgomery (2013)). Finally, some conclusions are brought in Section 6. 


\section{Structure of a multivariate gamma control chart based on the exact distribution}

Let $Y_{j}, j=1,2, \ldots, p$ be random variables following a gamma distribution with the shape parameter $\alpha_{j}-\alpha_{0}$ and the scale parameter $\beta$. Suppose $Y_{0}$ is a random variable that follows a gamma distribution with the shape parameter $\alpha_{0}$ and the scale parameter $\beta$. Assume that $Y_{0}, Y_{1}, \ldots, Y_{p}$ are mutually independent. Define $X_{j}=Y_{j}+Y_{0}, j=1,2, \ldots, p$. Then, $\boldsymbol{X}=\left(X_{1}, X_{2}, \ldots, X_{p}\right)$ follows a jointly p-variable gamma distribution. Each $X_{j}$ follows a gamma distribution marginally with the shape parameter $\alpha_{j}$ and the scale parameter $\beta$, where the covariance between two variables $\left(X_{i}, X_{j}\right)$ is equal to $\alpha_{0} \beta^{2}$. By the above definition, the multivariate gamma model is constructed with positive correlation and equal covariance between variables. The probability density function (pdf) of this multivariate gamma distribution can be written as follows:

$f_{\left(x_{1} \ldots . x_{p}\right)}\left(x_{1} \ldots . x_{p}\right)=\frac{1}{\left.\Gamma\left(\alpha_{0}\right) \prod_{i=1}^{p} \Gamma\left(\alpha_{i}-\alpha_{0}\right) \beta \Gamma_{i=1}^{p} \alpha_{i}\right)+\alpha_{0}(1-p)} \int_{0}^{\min \left(x_{1} \ldots . x_{p}\right)} e^{-\frac{\sum_{i=1}^{p} x_{i}+x_{0}(1-P)}{\beta}} x_{0}{ }^{\alpha_{0}-1} \prod_{i=1}^{p}\left(x_{i}-\right.$ $\left.x_{0}\right)^{\alpha_{i}-\alpha_{0}-1} d x_{0}, \quad x_{i}>0, \alpha_{i}>\alpha_{0}$.

The multivariate gamma distribution structure can be described with bellow example:

Suppose that a system has $p$ independent components so that $Y_{i}$, is longevity i-th item, and has a gamma distribution with the shape parameter $\alpha_{j}-\alpha_{0}$ and the scale parameter $\beta$. When i-th item fails, it is repaired or replaced by another item. Assume that $Y_{0}$ is the time of repaired or replaced of $i$-th item, and has a gamma distribution with the shape parameter $\alpha_{0}$ and the scale parameter $\beta$. In this case $X_{j}=Y_{j}+Y_{0}$ denotes the restart time of i-th item.

Define the statistic $D$ as follows:

$$
D=\sum_{j=1}^{p} X_{j}
$$

The probability density function of $D$ is given by:

$$
f_{D}(d)=\frac{e^{-d / p \beta} d^{\alpha_{0}+\alpha^{*}-1}}{p^{\alpha_{0} \beta^{\alpha_{0}+\alpha^{*}} \Gamma\left(\alpha_{0}+\alpha^{*}\right)}} F_{1,1}\left(\alpha^{*}, \alpha_{0}+\alpha^{*}, \frac{d(1-p)}{p \beta}\right),
$$

where, $\alpha^{*}=\left(\sum_{i=1}^{p} \alpha_{i}\right)-p \alpha_{0}$ and $F_{1,1}(a, b, z)$ denotes the hypergeometric function defined by

$$
F_{1,1}(a, b, z)=\frac{\Gamma(b)}{\Gamma(a) \Gamma(b-a)} \int_{0}^{1} t^{a-1}(1-t)^{b-a-1} \exp (t z) d t
$$

The probability density function of $D$ given in Equation (3) is derived based on the method of variable transformation in the Appendix.

Fixing Type-I error at $\alpha$, the lower and the upper control limits (LCL and UCL, respectively) are determined by solving the following two equations:

$$
\begin{gathered}
P(D<L C L)=\int_{0}^{L C L} f_{D}(t) \mathrm{d} t=\alpha / 2, \\
P(D>U C L)=\int_{U C L}^{\infty} f_{D}(t) \mathrm{d} t=\alpha / 2,
\end{gathered}
$$

Under this control chart, the out-of-control probability $\left(P_{\text {out }}^{0}\right)$ based on a single sample when the process is in-control is given by:

$$
P\left(D \leq L C L \mid \alpha_{i}=\alpha_{i 0}\right)+P\left(D \geq U C L \mid \alpha_{i}=\alpha_{i 0}\right) \text {. }
$$

The in-control ARL denoted by $\mathrm{ARL}_{0}$ is the expected number of subgroups to be examined until the process is declared to be out-of-control when the process is truly in control. It is given by:

$$
A R L_{0}=\frac{1}{P_{\text {out }}^{0}} .
$$

Now, suppose those shape parameters are changed from $\alpha_{i 0}$ to $\alpha_{i 1}=c \alpha_{i 0}, i=1,2, \ldots, p$ for a constant $c$. Then, the probability of the process being declared out-of-control based on the single sample when the process is changed is given by:

$$
P_{\text {out }}^{1}=P\left(D \leq L C L \mid \alpha_{i}=\alpha_{i 1}\right)+P\left(D \geq U C L \mid \alpha_{i}=\alpha_{i 1}\right) .
$$

Note that, the control limits are calculated when the process is in-control. The out-of-control ARL $\left(A R L_{1}\right)$ for the changing process is given as: 


$$
A R L_{1}=\frac{1}{P_{\text {out }}^{1}}
$$

\section{3- The efficiency evaluation of the chart}

In this section, the efficiency of the proposed control chart is evaluated using the ARL criterion. There are two types of ARL namely the in control $\left(\mathrm{ARL}_{0}\right)$ and the out-of-control $\left(\mathrm{ARL}_{1}\right) \mathrm{ARLs}$. The larger the value of the in-control and the smaller the value of the out-of-control ARL the better the performances of the chart are. Here, the $A_{R} L_{1} S$ of the chart are examined for a 3-variable and a 2-variable process when $\mathrm{ARL}_{0} \mathrm{~s}$ are fixed at 200 and 370 for each case. The results based on various parameter changes are shown in Tables 1 and 2, respectively. These results are obtained using MATLAB software.

Table 1. The $\mathrm{ARL}_{1}$ of the MG control chart based on the exact distribution of $D$ statistic, when $p=3$ and $\beta=4$.

\begin{tabular}{|c|c|c|c|c|c|c|c|c|}
\hline \multicolumn{3}{|c|}{$\boldsymbol{\alpha}=(3,3,3)$ and $\alpha_{0}=2$} & \multicolumn{2}{|c|}{$\boldsymbol{\alpha}=(9,7,9)$ and $\alpha_{0}=2$} & \multicolumn{2}{|c|}{$\boldsymbol{\alpha}=(5,1,3)$ and $\alpha_{0}=0.5$} & \multicolumn{2}{|c|}{$\boldsymbol{\alpha}=(2,1,2)$ and $\alpha_{0}=0.5$} \\
\hline $\mathrm{c}$ & $\begin{aligned} \mathrm{ARL}_{0} & =370 \\
L C L & =4.99 \\
U C L & =120.8\end{aligned}$ & $\begin{aligned} \mathrm{ARL}_{0} & =200 \\
L C L & =5.80 \\
U C L & =112.8\end{aligned}$ & $\begin{array}{l}\mathrm{ARL}_{0}=370 \\
L C L=44.50 \\
U C L=195.57\end{array}$ & $\begin{array}{c}\mathrm{ARL}_{0}=200 \\
L C L=47.5 \\
U C L=188.72\end{array}$ & $\begin{array}{l}\mathrm{ARL}_{0}=370 \\
L C L=8.90 \\
U C L=99.1\end{array}$ & $\begin{array}{c}\mathrm{ARL}_{0}=200 \\
L C L=9.9 \\
U C L=92.82\end{array}$ & $\begin{aligned} \mathrm{ARL}_{0} & =370 \\
L C L & =2.10 \\
U C L & =78.34\end{aligned}$ & $\begin{aligned} \mathrm{ARL}_{0} & =200 \\
L C L & =2.50 \\
U C L & =71.75\end{aligned}$ \\
\hline 0.7 & 20.28 & 15.13 & 10.34 & 7.38 & 27.26 & 18.58 & 38.52 & 26.25 \\
\hline 0.8 & 56.28 & 37.45 & 33.38 & 21.04 & 71.65 & 44.68 & 92.70 & 57.85 \\
\hline 0.9 & 163.76 & 96.56 & 132.28 & 73.37 & 187.63 & 107.19 & 209.66 & 120.15 \\
\hline 1 & 370.27 & 200.28 & 370.1 & 200 & 370.2 & 200.03 & 370.24 & 200.18 \\
\hline 1.1 & 457.16 & 246.91 & 283.63 & 171.06 & 407.41 & 220.36 & 446.93 & 238.30 \\
\hline 1.2 & 384.77 & 208.95 & 132.89 & 83.56 & 303.63 & 166.00 & 410.85 & 219.98 \\
\hline 1.3 & 283.17 & 157.94 & 62.02 & 40.04 & 202.22 & 110.93 & 338.54 & 181.54 \\
\hline 1.5 & 144.03 & 86.85 & 15.91 & 10.97 & 86.16 & 47.76 & 212.90 & 113.44 \\
\hline 1.7 & 84.50 & 48.40 & 5.40 & 4.05 & 37.88 & 21.64 & 131.24 & 69.45 \\
\hline 2.0 & 35.75 & 21.09 & 1.88 & 1.61 & 12.66 & 7.80 & 63.15 & 33.48 \\
\hline 2.2 & 20.84 & 12.62 & 1.30 & 1.20 & 6.88 & 4.53 & 39.09 & 20.99 \\
\hline 2.5 & 9.91 & 6.33 & 1.04 & 1.03 & 3.33 & 2.43 & 19.71 & 10.99 \\
\hline 2.7 & 6.40 & 4.24 & 1.01 & 1.00 & 2.31 & 1.80 & 12.92 & 7.46 \\
\hline 3.0 & 3.61 & 2.60 & 1.00 & 1.00 & 1.57 & 1.34 & 7.31 & 4.50 \\
\hline 3.2 & 2.65 & 1.92 & 1.00 & 1.00 & 1.32 & 1.19 & 5.24 & 3.38 \\
\hline 3.5 & 1.83 & 1.50 & 1.00 & 1.00 & 1.13 & 1.07 & 3.41 & 2.37 \\
\hline 3.7 & 1.52 & 1.03 & 1.00 & 1.00 & 1.07 & 1.03 & 2.69 & 1.95 \\
\hline
\end{tabular}

Table 2. The $\mathrm{ARL}_{1}$ of the MG control chart based on the exact distribution of $D$ statistic, when $p=2$ and $\beta=4$.

\begin{tabular}{|c|c|c|c|c|c|c|c|c|}
\hline \multicolumn{3}{|c|}{$\boldsymbol{\alpha}=(3,3)$ and $\alpha_{0}=2$} & \multicolumn{2}{|c|}{$\boldsymbol{\alpha}=(2,2)$ and $\alpha_{0}=0.5$} & \multicolumn{2}{|c|}{$\boldsymbol{\alpha}=(5,4)$ and $\alpha_{0}=2$} & \multicolumn{2}{|c|}{$\boldsymbol{\alpha}=(2,6)$ and $\alpha_{0}=0.5$} \\
\hline $\mathrm{c}$ & $\begin{array}{l}\mathrm{ARL}_{0}=370 \\
\left\{\begin{array}{c}L C L \\
\text { UCL }=81.16\end{array}\right.\end{array}$ & $\begin{array}{c}\mathrm{ARL}_{0}=200 \\
\left\{\begin{array}{l}L C L=3.10 \\
U C L=75.73\end{array}\right.\end{array}$ & $\begin{array}{l}\mathrm{ARL}_{0}=370 \\
\{C L=1.50 \\
U C L=59.59\end{array}$ & $\begin{array}{c}\mathrm{ARL}_{0}=200 \\
\left\{\begin{array}{l}L C L \\
U C L=54.75\end{array}\right.\end{array}$ & $\begin{array}{l}\mathrm{ARL}_{0}=370 \\
\left\{\begin{array}{l}L C L=7.80 \\
U C L=96.8\end{array}\right.\end{array}$ & $\begin{array}{l}\mathrm{ARL}_{0}=200 \\
\{C L=8.80 \\
U C L=91.62\end{array}$ & $\begin{array}{l}\mathrm{ARL}_{0}=370 \\
\left\{\begin{array}{cl}L C L & =7.60 \\
U C L & =81.36\end{array}\right.\end{array}$ & $\begin{aligned} \mathrm{ARL}_{0} & =200 \\
L C C L & =8.50 \\
U C L & =77.05\end{aligned}$ \\
\hline 0.7 & 26.63 & 19.68 & 44.64 & 32.17 & 27.57 & 18.91 & 32.98 & 22.03 \\
\hline 0.8 & 80.05 & 51.26 & 99.81 & 66.7 & 73.08 & 45.54 & 84.79 & 52.02 \\
\hline 0.9 & 198.78 & 114.98 & 212.64 & 128.98 & 193.85 & 109.92 & 212.17 & 119.77 \\
\hline 1 & 370.12 & 200.03 & 370 & 200.03 & 370.05 & 200.06 & 370.18 & 200.01 \\
\hline 1.1 & 415.78 & 224.11 & 448.71 & 222.12 & 367.09 & 204.01 & 331.52 & 183.44 \\
\hline 1.2 & 336.74 & 184.99 & 399.06 & 190.73 & 250.46 & 143.77 & 207.34 & 118.57 \\
\hline 1.3 & 247.1 & 137.64 & 307.95 & 146.32 & 158.03 & 92.56 & 121.89 & 71.54 \\
\hline 1.5 & 128.99 & 73.32 & 167.23 & 80.43 & 64.30 & 39.05 & 45.08 & 27.87 \\
\hline 1.7 & 96.01 & 40.13 & 91.47 & 45.07 & 28.42 & 18.01 & 19.17 & 12.54 \\
\hline 2.0 & 28.99 & 17.61 & 39.64 & 20.57 & 10.03 & 6.85 & 6.89 & 4.93 \\
\hline 2.2 & 17.19 & 10.81 & 23.98 & 12.97 & 5.69 & 4.11 & 4.08 & 3.09 \\
\hline 2.5 & 8.59 & 5.74 & 12.3 & 7.13 & 2.93 & 2.29 & 2.28 & 1.84 \\
\hline 2.7 & 5.77 & 4.02 & 8.35 & 5.07 & 2.10 & 1.74 & 1.73 & 1.49 \\
\hline 3.0 & 3.49 & 2.59 & 5.06 & 3.31 & 1.49 & 1.31 & 1.31 & 1.21 \\
\hline
\end{tabular}




\begin{tabular}{|l|ll|ll|ll|ll|}
\hline 3.2 & 2.65 & 2.06 & 3.82 & 2.62 & 1.28 & 1.17 & 1.18 & 1.11 \\
\hline 3.5 & 1.91 & 1.57 & 2.68 & 1.97 & 1.11 & 1.06 & 1.07 & 1.04 \\
\hline 3.7 & 1.61 & 1.38 & 2.2 & 1.69 & 1.06 & 1.03 & 1.03 & 1.01 \\
\hline
\end{tabular}

The conclusions made based on the results in Tables 1 and 2 are summarized as follow:

1 - It is observed that for $c>1$, when $c$ increases, the $A R L_{1}$ decreases for all cases.

2- $\quad \mathrm{ARL}_{1}$ decreases rapidly when $c>2$.

3- Most of the results show that if $c>1$, small changes of $c$ may not be detected $\left(\mathrm{ARL}_{1}>\mathrm{ARL}_{0}\right)$.

\section{Structure of the multivariate gamma control chart based on approximation}

In this section, two approximations are employed to obtain the control limits; the Satterthwaite approximation (Casella and Berger (2002)) and the Wilson-Hilferty (WH) approximation (Wilson and Hilferty (1931)).

\section{Case1: Satterthwaite approximation}

The Satterthwaite approximation is a suitable approach widely used today (Casella and Berger (2002)). Assume that the statistic $D$ in Equation (2) approximately follows a gamma distribution with the shape parameter $a$ and the scale parameter $b$. In the other words:

$$
D \sim \Gamma(a, b) .
$$

So, we have

$$
E(D)=a b,
$$

and

$$
\operatorname{Var}(D)=a b^{2} .
$$

Using the method of moment estimation (MME) and the Satterthwaite approximation,

$$
\begin{aligned}
E(D) & =E\left(\sum_{j=1}^{p} X_{j}\right)=a b, \\
\operatorname{Var}(D) & =\operatorname{Var}\left(\sum_{j=1}^{p} X_{j}\right)=a b^{2} .
\end{aligned}
$$

Thus, we have

$$
\sum_{j=1}^{p} E\left(X_{j}\right)=a b,
$$

and

$$
\sum_{j=1}^{p} \operatorname{Var}\left(X_{j}\right)+2 \sum \sum_{i<j} \operatorname{Cov}\left(X_{i} \cdot X_{j}\right)=a b^{2} .
$$

Hence, from (16) and (17) it can be concluded that

and

$$
a=\frac{\left(\sum_{i=1}^{p} \alpha_{i}\right)^{2}}{\sum_{i=1}^{p} \alpha_{i}+p(p-1) \alpha_{0}},
$$

$$
b=\beta\left[1+\frac{p(p-1) \alpha_{0}}{\sum_{i=1}^{p} \alpha_{i}}\right] .
$$

In the following, we assume that $b$ is known. From (11) it can be concluded that $\frac{2 D}{b}$ has a chi-squared distribution with $2 a$ degrees of freedom. In other words

$$
\frac{2 D}{b} \approx \chi_{2 a}^{2}
$$

Thus, the control limits of the chart with the probability of Type-I error $\alpha$ can be obtained as:

$$
\begin{aligned}
& P(D \geq U C L)=P\left(\frac{2 D}{b} \geq \frac{2 U C L}{b}\right)=P\left(\chi_{2 a}^{2} \geq \frac{2 U C L}{b}\right) \leq \alpha / 2, \\
& P(D \leq L C L)=P\left(\frac{2 D}{b} \leq \frac{2 L C L}{b}\right)=P\left(\chi_{2 a}^{2} \leq \frac{2 L C L}{b}\right) \leq \alpha / 2 .
\end{aligned}
$$

However, when the parameters $a$ and $b$ are unknown, their values can be estimated using an observed sample. Under the proposed control chart, the out-of-control probability $\left(P_{o u t}^{0}\right)$ based on a single sample when the process is incontrol is given by:

$$
P_{\text {out }}^{0}=P\left(D \leq L C L \mid a=a_{0}\right)+P\left(D \geq U C L \mid a=a_{0}\right)=P\left(\chi_{2 \mathrm{a}_{0}}^{2} \leq \frac{2 L C L}{b_{0}}\right)+P\left(\chi_{2 \mathrm{a}_{0}}^{2} \geq \frac{2 U C L}{b_{0}}\right) .
$$

Hence, 


$$
A R L_{0}=\frac{1}{P_{\text {out }}^{0}} .
$$

Now, suppose that the shape parameter of the gamma distribution is changed from $a_{0}$ to $a_{1}=c a_{0}$ (i.e one or all of the $\alpha_{i}$ s are changed), for a constant $c$. By (19), it can be concluded that scale parameter $b$ is shifted to $b_{1}$. Thus, the probability of the process being declared out-of-control based on the single sample when the process is changed is given by:

$$
P_{\text {out }}^{1}=P\left(D \leq L C L \mid a=a_{1}\right)+P\left(D \geq U C L \mid a=a_{1}\right)=P\left(\chi_{2 \alpha_{1}}^{2} \leq \frac{2 L C L}{b_{1}}\right)+P\left(\chi_{2 \alpha_{1}}^{2} \geq \frac{2 U C L}{b_{1}}\right) .
$$

As a result, the out-of-control ARL $\left(A R L_{1}\right)$ for the changing process is obtained as:

$$
A R L_{1}=\frac{1}{P_{\text {out }}^{1}} \text {. }
$$

\section{Case2: Normal approximation}

According to Wilson \& Hilferty (Wilson and Hilferty (1931)), the transformation $D^{*}=D^{\frac{1}{3}}$ approximately follows a normal distribution with the mean

and variance

$$
\mu_{D^{*}}=\frac{b^{\frac{1}{3}} \Gamma\left(a+\frac{1}{3}\right)}{\Gamma(a)}
$$

$$
\sigma_{D^{*}}^{2}=\frac{b^{\frac{2}{3}} \Gamma\left(a+\frac{2}{3}\right)}{\Gamma(a)}-\mu_{D^{*}}^{2} .
$$

Having $a_{0}$ the shape parameter of the gamma distribution when the process is in control, the traditional Shewharttype control limits using the above normal approximation can be written as follows:

and

$$
L C L=\mu_{D^{*}}-k \sigma_{D^{*}}=\frac{b_{0}^{\frac{1}{3}} \Gamma\left(a_{0}+\frac{1}{3}\right)}{\Gamma\left(a_{0}\right)}-3 \sqrt{\frac{b_{0}^{\frac{2}{3}} \Gamma\left(a_{0}+\frac{2}{3}\right)}{\Gamma\left(a_{0}\right)}-\mu_{D^{*}}{ }^{2}},
$$

$$
U C L=\mu_{D^{*}}+k \sigma_{D^{*}}=\frac{b_{0}^{\frac{1}{3}} \Gamma\left(a_{0}+\frac{1}{3}\right)}{\Gamma\left(a_{0}\right)}+3 \sqrt{\frac{b_{0}^{\frac{2}{3}} \Gamma\left(a_{0}+\frac{2}{3}\right)}{\Gamma\left(a_{0}\right)}-\mu_{D^{*}}{ }^{2}} .
$$

In Phase-I monitoring, the unknown parameters $a$ and $b$ are estimated based on the historical data set. Then, under normal approximation, the out-of-control probability when the process is in-control $P_{\text {out }}^{0}$ is obtained as

$$
P_{\text {out }}^{0}=P\left(D^{*}>U C L \mid a=a_{0}\right)+P\left(D^{*}<L C L \mid a=a_{0}\right)=2 \Phi(-3) \text {. }
$$

Thus, the ARL for the in-control process is given by

$$
A R L_{0}=\frac{1}{P_{\text {out }}^{0}}
$$

In what follows, we assume that the shape parameter of the gamma distribution is changed from $a_{0}$ to $a_{1}=c a_{0}$ (i.e one or all of the $\alpha_{i}$ s are changed) for a constant $c$. By (19), it can be concluded that the scale parameter $b$ is shifted to $b_{1}$. Then, the probability of the process being declared out-of-control based on the single sample is given by

$$
\begin{aligned}
P_{\text {out }}^{1} & =P\left(D^{*}>U C L \mid a=a_{1}\right)+P\left(D^{*}<L C L \mid a=a_{1}\right) \\
& =\Phi\left(L C L-\frac{b_{1}^{\frac{1}{3}} \Gamma\left(a_{1}+\frac{1}{3}\right)}{\Gamma\left(a_{1}\right)} /\left(\frac{b_{1} \frac{2}{3} \Gamma\left(a_{1}+\frac{2}{3}\right)}{\Gamma\left(a_{1}\right)}-\left(\frac{b_{1} \frac{1}{3} \Gamma\left(a_{1}+\frac{1}{3}\right)}{\Gamma\left(a_{1}\right)}\right)^{2}\right)^{\frac{1}{2}}\right) \\
& +1-\Phi\left(U C L-\frac{b_{1}^{\frac{1}{3}} \Gamma\left(a_{1}+\frac{1}{3}\right)}{\Gamma\left(a_{1}\right)} /\left(\frac{b_{1}^{\frac{2}{3}} \Gamma\left(a_{1}+\frac{2}{3}\right)}{\Gamma\left(a_{1}\right)}-\left(\frac{b_{1}^{\frac{1}{3}} \Gamma\left(a_{1}+\frac{1}{3}\right)}{\Gamma\left(a_{1}\right)}\right)^{2}\right)^{\frac{1}{2}}\right) .
\end{aligned}
$$

Note that, the control limits are calculated when the process is in-control. Besides, the out-of-control ARL for the shifted process is given by:

$$
A R L_{1}=\frac{1}{P_{\text {out }}^{1}}
$$

\section{Efficiency comparison}

Comparison among the efficiency of the three control charts proposed in Section 4 is demonstrated in this section. Here, the $A R L_{1} s$ of the charts are compared for a 3-variable and a 2-variable process when $\mathrm{ARL}_{0} \mathrm{~s}$ is fixed at 370 for each case. Moreover, $\mathrm{ARL}_{0}=200$ is considered when $p=3$. The results based on various parameter changes are shown in Tables 3-5. 
Table 3. The $\mathrm{ARL}_{1} \mathrm{~s}$ corresponding to the three proposed control charts when $p=3, \beta=4$ and $\mathrm{ARL}_{0}=370$.

\begin{tabular}{|c|c|c|c|c|c|c|c|c|c|}
\hline \multicolumn{4}{|c|}{$\boldsymbol{\alpha}=(9,7,9)$ and $\alpha_{0}=2$} & \multicolumn{3}{|c|}{$\boldsymbol{\alpha}=(5,1,3)$ and $\alpha_{0}=0.5$} & \multicolumn{3}{|c|}{$\boldsymbol{\alpha}=(4,4,4)$ and $\alpha_{0}=2$} \\
\hline$c$ & 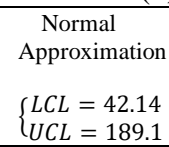 & 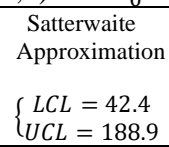 & $\begin{array}{c}\text { Exact } \\
\text { Distribution } \\
\left\{\begin{array}{c}L C L=44.5 \\
U C L=195.57\end{array}\right.\end{array}$ & $\begin{array}{c}\text { Normal } \\
\text { Approximation } \\
\left\{\begin{array}{c}L C L=7.76 \\
U C L=92.34\end{array}\right.\end{array}$ & $\begin{array}{l}\text { Satterwaite } \\
\text { Approximation } \\
\left\{\begin{array}{l}L C L=7.96 \\
U C L=91.89\end{array}\right.\end{array}$ & 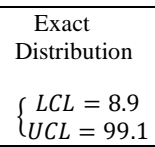 & $\begin{array}{c}\text { Normal } \\
\text { Approximation } \\
\left\{\begin{array}{c}L C L=8.99 \\
U C L=128.78\end{array}\right. \\
\end{array}$ & 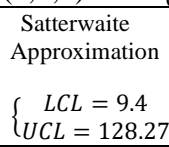 & $\begin{array}{c}\text { Exact } \\
\text { Distribution } \\
\left\{\begin{array}{c}L C L=11.1 \\
U C L=134.83\end{array}\right.\end{array}$ \\
\hline 0.7 & 12.3 & 11.98 & 10.34 & 31.53 & 29.27 & 27.26 & 27.56 & 25.4 & 19.58 \\
\hline 0.8 & 38.14 & 37.16 & 33.38 & 80.22 & 75.6 & 71.65 & 70.79 & 66.34 & 54.66 \\
\hline 0.9 & 141.06 & 138.77 & 132.28 & 202.28 & 196.34 & 187.63 & 185.16 & 179.15 & 163.13 \\
\hline 1 & 370.4 & 370.37 & 370.1 & 370.4 & 370.37 & 370.2 & 370.4 & 370.37 & 370 \\
\hline 1.1 & 253.27 & 251.39 & 283.63 & 350.57 & 348.76 & 407.41 & 400.71 & 399.28 & 416.86 \\
\hline 1.2 & 105.26 & 103.79 & 132.89 & 224.10 & 219.81 & 303.63 & 285.95 & 280.56 & 307.67 \\
\hline 1.3 & 45.50 & 44.78 & 62.02 & 132.69 & 129.14 & 202.22 & 182.79 & 177.71 & 207.48 \\
\hline 1.5 & 11.25 & 11.08 & 15.91 & 49.24 & 47.69 & 86.16 & 73.71 & 71.19 & 93.65 \\
\hline 1.7 & 4.04 & 4.00 & 5.39 & 20.82 & 20.19 & 37.88 & 31.70 & 30.56 & 43.71 \\
\hline 2.0 & 1.61 & 1.62 & 1.88 & 7.35 & 7.16 & 12.66 & 10.65 & 10.32 & 15.33 \\
\hline 2.2 & 1.21 & 1.21 & 1.30 & 4.28 & 4.19 & 6.87 & 5.86 & 5.71 & 8.29 \\
\hline 2.5 & 1.03 & 1.03 & 1.05 & 2.34 & 2.31 & 3.33 & 2.91 & 2.85 & 3.84 \\
\hline 2.7 & 1.00 & 1.00 & 1.01 & 1.76 & 1.74 & 2.32 & 2.06 & 2.03 & 2.57 \\
\hline 3.0 & 1.00 & 1.00 & 1.00 & 1.32 & 1.31 & 1.57 & 1.44 & 1.42 & 1.66 \\
\hline 3.2 & 1.00 & 1.00 & 1.00 & 1.18 & 1.17 & 1.33 & 1.24 & 1.23 & 1.36 \\
\hline 3.5 & 1.00 & 1.00 & 1.00 & 1.07 & 1.06 & 1.13 & 1.09 & 1.08 & 1.14 \\
\hline 3.7 & 1.00 & 1.00 & 1.00 & 1.03 & 1.03 & 1.07 & 1.04 & 1.04 & 1.07 \\
\hline
\end{tabular}

Table 4. The $\mathrm{ARL}_{1} \mathrm{~s}$ corresponding to the three proposed control charts when $p=2, \beta=4$ and $\mathrm{ARL}_{0}=370$.

\begin{tabular}{|c|c|c|c|c|c|c|c|c|c|}
\hline \multicolumn{4}{|c|}{$\boldsymbol{\alpha}=(2,2)$ and $\alpha_{0}=0.5$} & \multicolumn{3}{|c|}{$\boldsymbol{\alpha}=(4,4)$ and $\alpha_{0}=2$} & \multicolumn{3}{|c|}{$\boldsymbol{\alpha}=(2,9)$ and $\alpha_{0}=0.5$} \\
\hline$c$ & $\begin{array}{c}\text { Normal } \\
\text { Approximation } \\
\left\{\begin{array}{l}L C L=1.09 \\
U C L=56.62\end{array}\right.\end{array}$ & $\begin{array}{c}\text { Satterwaite } \\
\text { Approximation } \\
\left\{\begin{array}{l}L C L=1.28 \\
U C L=56.2\end{array}\right.\end{array}$ & $\begin{array}{c}\text { Exact } \\
\text { Distribution } \\
L C L=1.5 \\
\text { UCL }=59.59\end{array}$ & $\begin{array}{c}\text { Normal } \\
\text { Approximation } \\
\left\{\begin{array}{l}L C L=5.18 \\
U C L=89.81\end{array}\right.\end{array}$ & $\begin{array}{c}\text { Satterwaite } \\
\text { Approximation } \\
\left\{\begin{array}{lll}L C L & =58 \\
U C L & 89.67\end{array}\right.\end{array}$ & $\begin{array}{c}\text { Exact } \\
\text { Distribution } \\
L C L=5.9 \\
\text { UCL }=91.95\end{array}$ & $\begin{array}{c}\text { Normal } \\
\text { Approximation } \\
\left\{\begin{array}{l}L C L=13.48 \\
U C L=97.33\end{array}\right.\end{array}$ & $\begin{array}{c}\text { Satterwaite } \\
\text { Approximation } \\
\left\{\begin{array}{l}L C L=13.65 \\
U C L=97.3\end{array}\right.\end{array}$ & $\begin{array}{c}\text { Exact } \\
\text { Distribution } \\
\begin{cases}L C L=13.9 \\
U C L=98.66\end{cases} \end{array}$ \\
\hline 0.7 & 56.47 & 48.09 & 44.64 & 34.84 & 31.71 & 29.3 & 25.39 & 24.17 & 23.68 \\
\hline 0.8 & 120.9 & 107.91 & 99.81 & 85.5 & 79.53 & 76.29 & 69.36 & 66.52 & 65.44 \\
\hline 0.9 & 239.17 & 227.05 & 212.64 & 205.13 & 197.98 & 196.94 & 194.12 & 189.88 & 186.86 \\
\hline 1 & 370.4 & 370.37 & 370 & 370.4 & 370.39 & 370.1 & 370.4 & 370.37 & 370 \\
\hline 1.1 & 396.55 & 395.61 & 448.71 & 383.99 & 383.05 & 383.78 & 290.01 & 288.02 & 304.67 \\
\hline 1.2 & 323.5 & 320.45 & 399.06 & 274.8 & 269.8 & 285.07 & 150.37 & 147.76 & 163.02 \\
\hline 1.3 & 236.71 & 231.09 & 307.95 & 177.52 & 172.55 & 182.85 & 76.8 & 75.13 & 84.59 \\
\hline 1.5 & 120.81 & 116.032 & 167.23 & 74.08 & 71.48 & 80.23 & 23.88 & 23.34 & 26.47 \\
\hline 1.7 & 64.10 & 61.44 & 91.47 & 33.43 & 32.22 & 37.42 & 9.43 & 9.24 & 10.35 \\
\hline 2.0 & 27.57 & 26.43 & 39.64 & 12.09 & 11.7 & 13.74 & 3.44 & 3.40 & 3.68 \\
\hline 2.2 & 16.86 & 16.20 & 23.98 & 6.91 & 6.72 & 7.81 & 2.18 & 2.16 & 2.30 \\
\hline 2.5 & 8.92 & 8.61 & 12.3 & 3.53 & 3.46 & 3.91 & 1.41 & 1.41 & 1.46 \\
\hline 2.7 & 6.2 & 6.01 & 8.35 & 2.5 & 2.46 & 2.72 & 1.20 & 1.20 & 1.22 \\
\hline 3.0 & 3.9 & 3.82 & 5.06 & 1.71 & 1.69 & 1.81 & 1.06 & 1.06 & 1.07 \\
\hline 3.2 & 3.04 & 2.97 & 3.82 & 1.43 & 1.41 & 1.50 & 1.02 & 1.02 & 1.03 \\
\hline 3.5 & 2.22 & 2.18 & 2.68 & 1.20 & 1.19 & 1.23 & 1.00 & 1.00 & 1.00 \\
\hline 3.7 & 1.88 & 1.85 & 2.20 & 1.11 & 1.12 & 1.13 & 1.00 & 1.00 & 1.00 \\
\hline
\end{tabular}


Table 5. The $\mathrm{ARL}_{1}$ s corresponding to the three proposed control charts when $p=3, \beta=4$ and $\mathrm{ARL}_{0}=200$.

\begin{tabular}{|c|c|c|c|c|c|c|c|c|c|}
\hline \multicolumn{4}{|c|}{$\boldsymbol{\alpha}=(9,7,9)$ and $\alpha_{0}=2$} & \multicolumn{3}{|c|}{$\boldsymbol{\alpha}=(5,1,3)$ and $\alpha_{0}=0.5$} & \multicolumn{3}{|c|}{$\boldsymbol{\alpha}=(4,4,4)$ and $\alpha_{0}=2$} \\
\hline$C$ & $\begin{array}{c}\text { Normal } \\
\text { Approximation } \\
\left\{\begin{array}{c}L C L=44.73 \\
U C L=183.25\end{array}\right.\end{array}$ & $\begin{array}{c}\text { Satterwaite } \\
\text { Approximation } \\
\left\{\begin{array}{c}L C L=45.07 \\
U C L=181.89\end{array}\right.\end{array}$ & $\begin{array}{c}\text { Exact } \\
\text { Distribution } \\
L C L=47.5 \\
C L L=188.72\end{array}$ & $\begin{array}{c}\text { Normal } \\
\text { Approximation } \\
L C L=8.74 \\
U C L=87.52\end{array}$ & 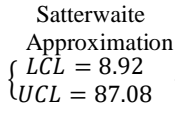 & $\begin{array}{c}\text { Exact } \\
\mathrm{n} \quad \begin{array}{c}\text { Distribution } \\
L C L=9.9 \\
U C L=92.82\end{array}\end{array}$ & $\begin{array}{c}\text { Normal } \\
\text { Approximation } \\
\left\{\begin{array}{c}L C L=10.36 \\
U C L=121.29\end{array}\right.\end{array}$ & $\begin{array}{c}\text { Satterwaite } \\
\text { Approximation } \\
\left\{\begin{array}{c}L C L=10.64 \\
U C L=121.27\end{array}\right.\end{array}$ & $\begin{array}{c}\text { Exact } \\
\text { Distribution } \\
L C L=12.4 \\
U C L=127.12\end{array}$ \\
\hline 0.7 & 9.08 & 8.90 & 7.38 & 21.61 & 20.37 & 18.5 & 19.34 & 18.12 & 14.09 \\
\hline 0.8 & 25.49 & 24.99 & 21.04 & 50.6 & 48.27 & 44.68 & 45.47 & 43.16 & 35.33 \\
\hline 0.9 & 84.23 & 83.16 & 73.37 & 116.8 & 114.06 & 107.19 & 108.06 & 105.24 & 94.43 \\
\hline 1 & 200.1 & 200 & 200.03 & 200 & 200 & 200.03 & 200 & 200 & 200.03 \\
\hline 1.1 & 141.16 & 140.33 & 171.06 & 189.98 & 189.45 & 220.36 & 213.87 & 213.60 & 230.24 \\
\hline 1.2 & 61.91 & 61.23 & 83.56 & 125.15 & 123.45 & 166.00 & 155.26 & 153.24 & 174.62 \\
\hline 1.3 & 28.14 & 27.80 & 40.04 & 76.21 & 74.71 & 110.93 & 100.96 & 98.93 & 119.3 \\
\hline 1.5 & 7.73 & 7.65 & 10.97 & 38.26 & 29.19 & 47.76 & 42.27 & 41.25 & 54.88 \\
\hline 1.7 & 3.09 & 3.07 & 4.05 & 13.4 & 13.12 & 21.64 & 19.1 & 18.63 & 26.26 \\
\hline 2.0 & 1.42 & 1.42 & 1.61 & 5.18 & 5.10 & 7.8 & 6.99 & 6.85 & 9.73 \\
\hline 2.2 & 1.13 & 1.13 & 1.20 & 3.21 & 3.17 & 4.53 & 4.1 & 4.032 & 5.52 \\
\hline 2.5 & 1.02 & 1.02 & 1.03 & 1.91 & 1.89 & 2.43 & 2.24 & 2.21 & 2.79 \\
\hline 2.7 & 1.00 & 1.00 & 1.00 & 1.51 & 1.50 & 1.80 & 1.68 & 1.67 & 1.99 \\
\hline 3.0 & 1.00 & 1.00 & 1.00 & 1.21 & 1.20 & 1.34 & 1.27 & 1.27 & 1.40 \\
\hline 3.2 & 1.00 & 1.00 & 1.00 & 1.11 & 1.11 & 1.19 & 1.14 & 1.14 & 1.21 \\
\hline 3.5 & 1.00 & 1.00 & 1.00 & 1.02 & 1.02 & 1.07 & 1.05 & 1.04 & 1.07 \\
\hline 3.7 & 1.00 & 1.00 & 1.00 & 1.01 & 1.01 & 1.03 & 1.02 & 1.02 & 1.03 \\
\hline
\end{tabular}

The results in Tables 3-5 show that when $c<1$, the control chart based on the exact distribution has smaller ARL 1 values. For example, in the case $\mathrm{ARL}_{0}=370, \boldsymbol{\alpha}=(9,7,9)$ and $c=0.7$ (the first column of Table 3 ), the value of $\mathrm{ARL}_{1}$ for the exact distribution is 10.34 , while it is 11.98 for the Satterthwaite approximation and 12.3 for the normal approximation. In addition, when $c>1$, the control chart based on the Satterthwaite approximation has smaller ARL values; thus it can detect the changes earlier. Besides, based on the results in the above tables one can conclude that for large changes $(c>2.5)$, the three charts have almost an equal performance in terms of the out-of-control ARL. So, can conclude that the Satterthwaite approximation and the normal approximation are good and appropriate approximations.

\section{Conclusions and remarks}

In the present article, a multivariate gamma distribution with positive correlations was introduced. Then, by defining a statistic, three control charts were proposed to monitor processes modeled by this distribution. The first control chart was based on the exact distribution of the defined statistic, the second control chart was based on the Satterthwaite approximation, and the third was based on the Wilson-Hilferty approximation. As the Wilson-Hilferty approximation is used to approximate a gamma distribution by a normal distribution, a Shewhart control chart was constructed based on this approximation. The efficiency of these charts was compared in terms of out-of-control ARL, when $\mathrm{ARL}_{0}$ remains constant. It was observed that whenever the magnitude of the parameter shifts $c<1$, the control chart based on the exact distribution had smaller $\mathrm{ARL}_{1} \mathrm{~s}$, while for $c>1$, the control chart based on Satterthwaite approximation showed smaller values of $\mathrm{ARL}_{1} \mathrm{~s}$. Besides, it was observed that for large changes $(c>2.5)$, all proposed charts had almost equal $\mathrm{ARL}_{1}$ s; i.e. they had the same performance.

\section{Acknowledgments}

The authors sincerely thank the editor and anonymous reviewers for their constructive suggestions and comments, which helped us to improve the presentation of the article. 


\section{References}

Aslam, M., Khan, N. \& Jun, C.H. (2016). A control chart using belief information for a gamma distribution. Operations Research and Decisions, 26(4), 5-19.

1. Aslam, M., Srinivasa Rao, G., Ahmad, L. \& Jun, C.H. (2017). A control chart for multivariate Poisson distribution using repetitive sampling. Journal of Applied Statistics, 44(1), 123-136.

2. Ali Raza, M. \& Aslam, M. (2018). Design of control charts for multivariate Poisson distribution using generalized multiple dependent state sampling. Quality Technology \& Quantitative Management, 16(6),629650.

3. Aksoy, H. (2000). Use of gamma distribution in hydrological analysis. Turkish Journal of Engineering and Environmental Sciences, 24(6), 419-428.

4. Agarwal, S.K. \& Kalla, S.L. (1996). A generalized gamma distribution and its application in reliability. Communications in Statistics - Theory and Methods, 25(1), $201-210$.

5. Cozzucoli, P. C. \& Marozzi, M. (2018). Monitoring multivariate Poisson processes: A review and some new results. Quality Technology \& Quantitative Management, 15(1), 53-68.

6. Casella, G. \& Berger, R.L. (2002). Statistical Inference (Vol.2). Pacific Grove, CA: Duxbury.

7. Chiu, J. E. \& Kuo, T.I. (2007). Attribute control chart for multivariate Poisson distribution. Communications in Statistics-Theory and Methods, 37(1), 146-158.

8. Derya, K. \& Canan, H. (2012). Control Charts for Skewed Distributions: Weibull, Gamma, and Lognormal. Advances in Methodology \& Statistics. Metodoloskizvezki, 9(2), 95-106.

9. Khan, N., Aslam, M., Ahma, L. \& Jun, C.H. (2017). A control chart for gamma distributed variables using repetitive sampling scheme. Pakistan Journal of Statistics and Operation Research, 13(1), 47-61.

10. Mori, S., Nakata, D. \& Kaneda, T. (2015). An Application of Gamma Distribution to the Income Distribution and the Estimation of Potential Food Demand Functions. Modern Economy, 6(9), 1001-1017.

11. Montgomery, D.C. (2013). Design \& Analysis of Experiments. John Wiley \& Sons.

12. Tan, C.M., Raghavan, N. \& Roy, A. (2007). Application of gamma distribution in electro migration for submicron interconnects. Journal of Applied Physics, 102, 103703.

13. Wilson, E.B. \& Hilferty, M.M. (1931). The Distribution of Chi-Squares. Proceedings of the National Academy of Sciences of the United States of America, 17(12), 684-688.

14. Zhang, C.W., Xie, M., Liu, J.Y. \& Goh, T.N. (2007). A control chart for the Gamma distribution as a model of time between events. International Journal of Production Research, 45(23), 5649-5666.

\section{Appendix}

\section{Calculating the exact distribution of the $D$ statistic}

Based on the definition of $X_{i} \mathrm{~s}$, we can write

$$
D=\sum_{i=1}^{p} X_{i}=\sum_{i=1}^{p}\left(Y_{i}+Y_{0}\right)=\left(\sum_{i=1}^{p} Y_{i}\right)+p Y_{0} .
$$

Suppose that $T=\sum_{i=1}^{p} Y_{i}$ and $Z=p Y_{0}$. Then, we have

$$
T \sim \Gamma\left(\sum_{i=1}^{p} \alpha_{i}-p \alpha_{0}, \beta\right), Z \sim \Gamma\left(\alpha_{0}, p \beta\right),
$$

where $T$ and $Z$ are independent.

Let $D=T+Z$ and $W=T$. then we have $T=W$ and $Z=D-W$. Using the change of variables technique, the joint probability density function of $D$ and $W$ is obtained in the following form

$$
\begin{aligned}
& f_{D, W}(d, w)=f_{Z, T}(d-w, w)=f_{Z}(d-w) f_{T}(w) \\
& \quad=\frac{1}{\Gamma\left(\alpha_{0}\right) \Gamma\left(\alpha^{*}\right)(p \beta)^{\alpha_{0} \beta^{\alpha^{*}}}}(d-w)^{\alpha_{0}-1} e^{-\frac{d-w(1-p)}{p \beta}} w^{\alpha^{*}-1}, w>0, d>w,
\end{aligned}
$$

where $\alpha^{*}=\left(\sum_{i=1}^{p} \alpha_{i}\right)-p \alpha_{0}$.

By integrating of the above function concerning $w$ and using the change of variable $=\frac{w}{d}$, it can be shown that the probability density function of $D$ is the same as the mentioned probability density function in Equation (3). 
